\title{
Lenalidomide, celecoxib, and azacitidine therapy for blastic plasmocytoid dendritic cell neoplasm: a case report
}

This article was published in the following Dove Press journal:

OncoTargets and Therapy

7 September 2016

Number of times this article has been viewed

\author{
Marta Garcia-Recio ${ }^{1,2}$ \\ Jordi Martinez-Serra ${ }^{1,2}$ \\ Leyre Bento ${ }^{1,2}$ \\ Rafael Ramos ${ }^{2,3}$ \\ Jordi Gines ${ }^{4}$ \\ Jaime Daumal ${ }^{5}$ \\ Antonia Sampol ${ }^{1,2}$ \\ Antonio Gutierrez ${ }^{1,2}$
}

'Service of Hematology, ${ }^{2}$ Instituto de Investigación Sanitaria de Palma (IdISPa), ${ }^{3}$ Service of Pathology, ${ }^{4}$ Pharmacy Department, ${ }^{5}$ Service of Nuclear Medicine, Son Espases University Hospital, Palma de Mallorca, Spain
Correspondence: Antonio Gutierrez Service of Hematology, Son Espases University Hospital, Carretera de Valldemossa, 70, Palma de Mallorca 07010, Spain Tel +3487 I 205000 ext 65 II 5 or 75934 Email antoniom.gutierrez@ssib.es

\begin{abstract}
Blastic plasmocytoid dendritic cell neoplasm is characterized by aggressive behavior with a tendency for systemic dissemination and a predilection for skin, lymph nodes, soft tissues, peripheral blood, or bone marrow. It usually occurs in elderly patients with a mean age between 60 and 70 years. Despite initial response to chemotherapy, the disease regularly relapses with a short median overall survival. Better outcomes have been reported with high-dose acute leukemia-like induction chemotherapy followed by consolidation with allogeneic hematopoietic stem cell transplantation. However, elderly patients are not candidates for intensive therapy or allogeneic stem cell transplantation. So, new active and tolerable drugs are needed. Our case illustrates that one cycle of lenalidomide and celecoxib provides at least a partial cutaneous and hematologic response, but this regimen was discontinued due to toxicity and followed by a consolidation/maintenance phase with azacitidine, thus achieving a final complete response with a much higher than expected progression-free and overall survival in an elderly patient with comorbidities. This information may be useful in the design of treatment approaches for elderly patients with blastic plasmocytoid dendritic cell neoplasm. However, it should be confirmed in clinical trials as well as by optimizing the induction and extending the consolidation/maintenance period to avoid early relapses after discontinuation and improve progression-free survival.

Keywords: blastic plasmocytoid dendritic cell neoplasm, elderly, immunomodulatory, COX-2, hypomethylating
\end{abstract}

\section{Introduction}

Blastic plasmocytoid dendritic cell neoplasm (BPDCN) is a rare and aggressive type of myeloid malignancy characterized by the clonal proliferation of precursors of $\mathrm{CD}_{123}{ }^{+} \mathrm{CD} 56{ }^{+}$plasmocytoid dendritic cells (DCs). ${ }^{1} \mathrm{BPDCN}$ is characterized by aggressive behavior with a tendency to systemic dissemination and predilection for skin, lymph nodes, soft tissues, peripheral blood, or bone marrow. It usually occurs in elderly patients with a mean age between 60 and 70 years. ${ }^{2}$ Despite an initial response to chemotherapy, the disease regularly relapses and has a short median overall survival (OS).

Currently, there is no standard treatment approach for BPDCN, and the optimal therapy remains to be defined. Better outcomes have been reported in younger patients started with high-dose acute lymphoblastic leukemia and acute myeloid leukemia (AML)-like induction chemotherapy followed by consolidation with allogeneic hematopoietic stem cell transplantation (HSCT), ${ }^{3}$ but the majority of patients are too old or unfit to undergo this intensive therapeutic approach, so alternative strategies with more effective and less toxic drugs should be considered. 
We present a case of BPDCN in an unfit 79-year-old woman who was not a candidate for intensive therapy and who responded to a combination therapy of lenalidomide and celecoxib, which was followed by azacitidine treatment. We discuss the rationale behind the use of these new drugs in BPDCN.

\section{Case presentation}

A 79-year-old woman with only a history of toxic thyroid adenoma (undergoing treatment with carbimazol) consulted the dermatologist in January 2014 because of an 8-month history of small, violet, cutaneous abdominal lesions that had spread to the lower extremities, neck, armpits, and scalp (Figure 1A). She presented with asthenia, and the physical examination also revealed $2 \mathrm{~cm}$ left supraclavicular and right laterocervical lymphadenopathies. Complete blood count showed a bicytopenia with moderate anemia $(8.92 \mathrm{~g} / \mathrm{dL})$ and thrombocytopenia of $44.500 / \mu \mathrm{L}$. Biochemistry also reflected mild renal (creatinine $1.35 \mathrm{mg} / \mathrm{dL}$ ) and hepatic (total bilirubin $1.7 \mathrm{mg} / \mathrm{dL}$ ) failures. Immunoglobulins were normal, but $\beta 2$-microglobulin was elevated $(12,900 \mu \mathrm{g} / \mathrm{L})$. Human immunodeficiency virus, hepatitis, and herpes virus serologies were negative except for cytomegalovirus (IgM).

In February 2014, the cutaneous biopsy revealed a diffuse infiltration of the dermis by medium-sized blastic cells with ovoid nuclei, agranular cytoplasm, and multiple mitoses. There was no epidermal invasion. These cells were positive for CD4, CD56, CD68, and TdT, but negative for cytotoxic granules and myeloperoxidase, CD30, EBER, or CD20. The results were compatible with a diagnosis of BPDCN. Bone marrow biopsy also showed that $31 \%$ of blasts had an agranular cytoplasm and a normal cytogenetic profile (46XX). Positron emission tomography-computed tomography (PET/CT) showed metabolically active nodal (supra and infradiaphragmatic) and extranodal (skin and spleen) disease. The study also revealed diffuse bone and right breast involvement.

With the final diagnosis of hematodermic BPDCN in an elderly patient with comorbidities, who was not a candidate for intensive chemotherapy or allogeneic HSCT, we recommended a low-toxicity therapeutic approach consisting of an induction therapy with one to three cycles of lenalidomide
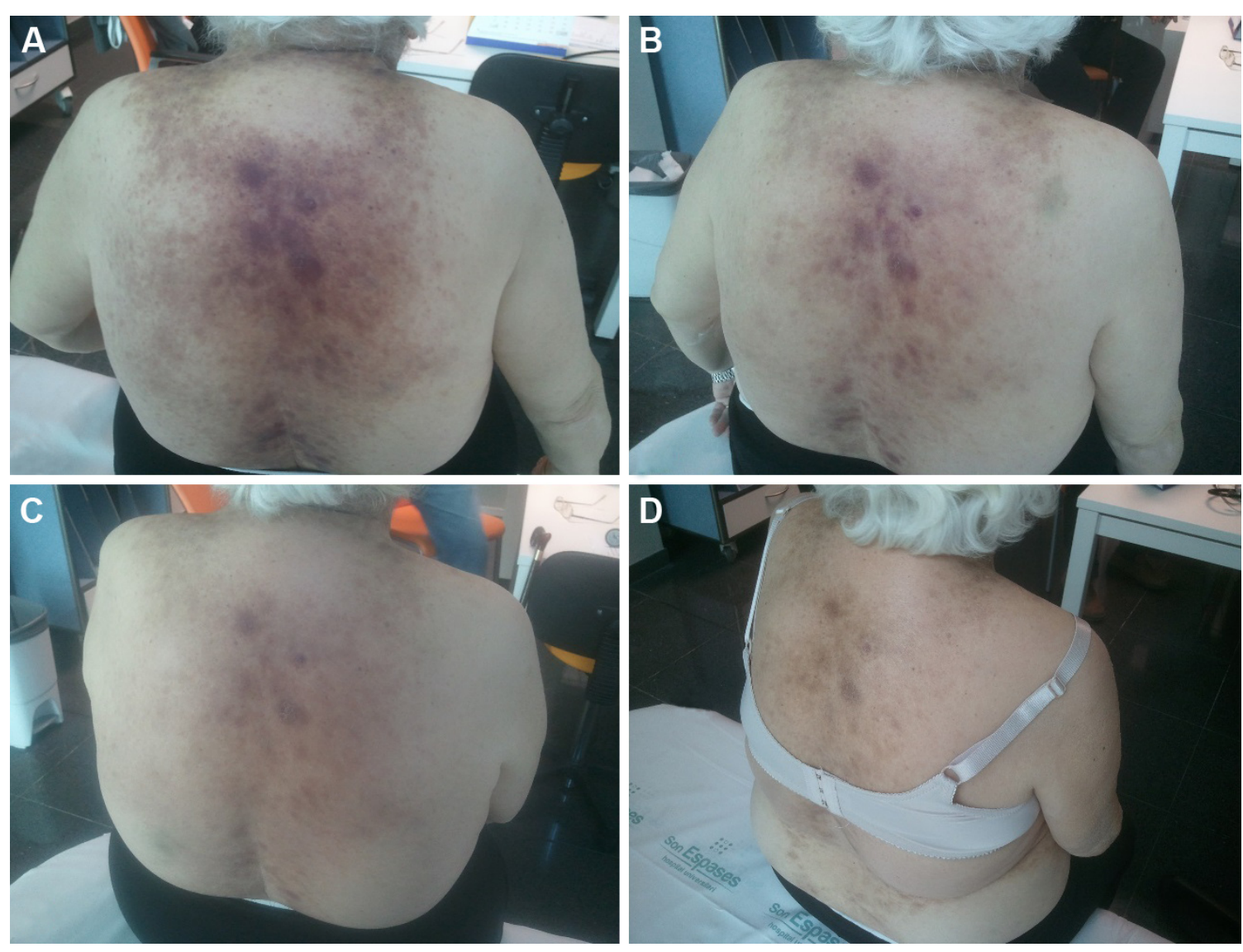

Figure I Skin lesions reflecting initial outcome after lenalidomide/celecoxib.

Notes: (A) Before treatment. (B) After I week of treatment. (C) After 3 weeks of treatment. (D) After six courses of azacitidine. 
$20 \mathrm{mg}$ for three of every 4 weeks combined with celecoxib $200 \mathrm{mg}$ every 12 hours, followed by maintenance therapy with azacitidine.

The rationale of this therapy was based on the preliminary French experience of the clinical activity of 5-azacytidine reported by Laribi et al, ${ }^{4}$ who demonstrated a partial cutaneous response and hematologic stabilization in two elderly BPDCN patients. However, to achieve a prior complete response (CR), we aimed to administer an induction regimen with low toxicity after considering the patient's age and comorbidities.

A preclinical study in a mouse xenograft model of BPDCN was reported in 2011 showing promising activity of lenalidomide. The authors attributed this efficacy to the antiangiogenic properties of the drug as well as modification of cytokines production. ${ }^{5}$ At the same time, celecoxib, a selective cyclooxygenase-2 (COX-2) inhibitor, has demonstrated activity against AML and chronic myeloid leukemia, ${ }^{6}$ so we decided to try a combination of both drugs for initial debulking prior to azacitidine therapy.

The patient began induction therapy in March 2014. During the first 15 days of treatment, the skin lesions progressively reduced in size (Figure 1B and C), and total bilirubin and creatinine also normalized, thus leading to improved patient performance status. On Day +19 , our patient attended a preprogrammed visit and was found to have renal (creatinine $2.6 \mathrm{mg} / \mathrm{dL}$ ) and hepatic failure (total bilirubin $17 \mathrm{mg} / \mathrm{dL}$; direct bilirubin $13.6 \mathrm{mg} / \mathrm{dL}$ ) and a worse general condition. Because of this situation after the first cycle, we stopped both lenalidomide and celecoxib and only started supportive care measures. However, on Day +35 , our patient had recovered performance status and renal function and even showed a significant improvement in liver function (total bilirubin $2.2 \mathrm{mg} / \mathrm{dL}$ ). At this point, the skin lesions had regressed and the hematological alterations improved (hemoglobin $10.6 \mathrm{~g} / \mathrm{dL}$ ). So, the patient had obtained at least a partial cutaneous clinical and hematological response with one cycle of the lenalidomide-celecoxib regimen.

On Day +49 after onset of therapy (April 2014), we started consolidation/maintenance therapy with azacitidine $75 \mathrm{mg} / \mathrm{m}^{2}$ for 7 days every 28 days. After six cycles, a $\mathrm{PET} / \mathrm{CT}$ and bone marrow aspiration showed a complete metabolic response and full immunophenotypic disappearance of the malignant clone (Figures 1D and 2). The patient continued with this therapy until the completion of twelve cycles in February 2015. At that point of time, the patient was asymptomatic, with no cutaneous evidence of disease and a normal complete blood count, and so we proceeded to discontinue the therapy.

Three months after discontinuation (May 2015), the patient presented with a cutaneous lesion on the forehead and had severe neutropenia (530 neutrophils/ $\mu \mathrm{L})$ and mild thrombocytopenia $(113,000$ platelets/ $\mu \mathrm{L})$. A myelogram revealed $7 \%$ agranular blastic population and an immunophenotype compatible with blastic plasmocytoid DCs. This time, the disease progressed rapidly and the patient did not respond
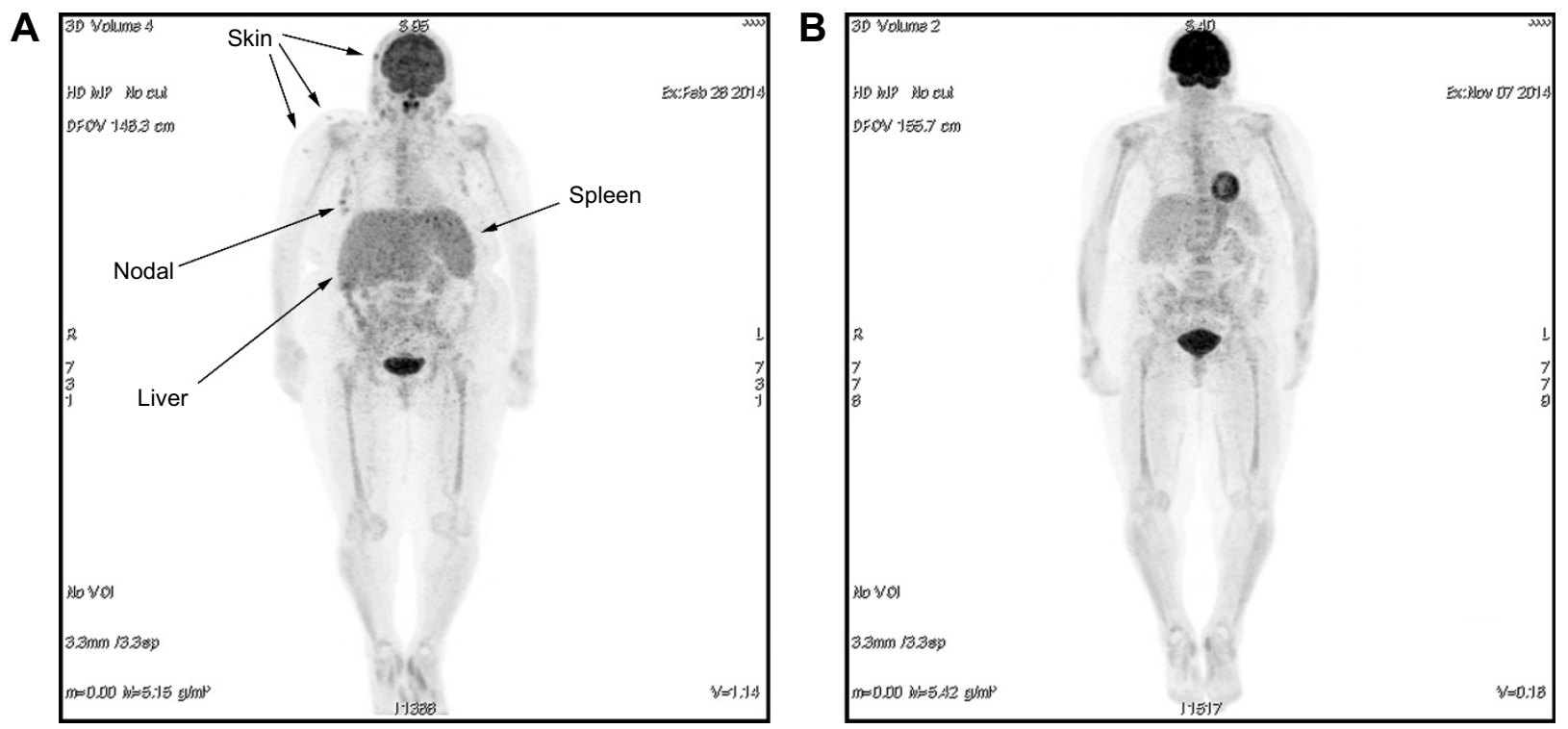

Figure 2 PET/CT evolution of cutaneous, nodal, hepatic, and splenic involvement.

Notes: (A) At diagnosis (black arrows pointing to skin, nodal, hepatic, and splenic involvement). (B) After six courses of azacitidine showing metabolic complete response. Abbreviation: PET/CT, positron emission tomography-computed tomography. 
to two additional cycles of azacitidine, dying as a result of multiorgan failure 17 months after the onset of therapy.

Written informed consent was obtained for the use of clinical data, participation in laboratory tests, and medical images.

\section{Discussion}

BPDCN is a great challenge especially in elderly patients who are not candidates for intensive therapy or allogeneic stem cell transplantation. So, new active and tolerable drugs are needed. Our case illustrates the activity of lenalidomide, celecoxib, and azacitidine in providing CR with a progression-free survival and OS higher than that expected in a patient of this age with this diagnosis.

Lenalidomide is a known therapeutic option in myeloid malignancies such as myelodysplastic syndromes and AML. It acts through immunomodulatory, anti-inflammatory, antiangiogenic, and antiproliferative mechanisms. It enhances antigen uptake by antigen-presenting cells and potentiates immune responses by restoring DC function, inhibiting regulatory $\mathrm{T}$-cell activity, and activating natural killer cells and T-cells by boosting the production of interferon gamma and interleukin-2., ${ }^{7,8}$ Furthermore, lenalidomide has shown activity in a preclinical mouse model of BPDCN. ${ }^{5}$

COX-2 is involved in the development and progression of a range of cancers metabolizing arachidonic acid to produce prostaglandin E2, which in turn induces tumor angiogenesis ${ }^{9}$ as well as modulating Th1 and Th2 immune responses, thus blocking antitumor immunity. ${ }^{10} \mathrm{COX}-2$ inhibitors, such as celecoxib, act through the prostaglandin E2-cyclic adenosine monophosphate-protein kinase C-nuclear factor kappa B $(\mathrm{NF}-\kappa \mathrm{B})$ pathway regulating the expression of multidrug resistance-associated protein-1 and other cellular proteins abolishing drug resistance..$^{11,12}$

Furthermore, a recent study showed an aberrant activation of the NF- $\kappa$ B pathway as a key molecular event in BPDCN, suggesting its blockade as a therapeutic target. ${ }^{13}$ These authors reported the activity of a NF- $\mathrm{KB}$ inhibitor such as bortezomib, but also lenalidomide and celecoxib, may inhibit the NF-кB pathway.

A combination of thalidomide or lenalidomide with celecoxib has shown activity in several malignancies such as prostate cancer, ${ }^{14}$ sarcomas, ${ }^{15}$ or myeloma. ${ }^{16}$ However, the combination regimen may potentially be toxic, as in our patient, and so a special monitoring of renal and liver functions as well as hematologic toxicity is needed. In our case, cutaneous lesions resolved after one cycle of this combination therapy, ie, it showed clinical activity (Figure 1C).
However, the renal and hepatic toxicity observed in the patient prompted us to discontinue therapy and begin a consolidation/maintenance therapy with azacitidine.

Azacitidine is a pyrimidine nucleoside analog of cytidine that acts as a hypomethylating drug that upregulates suppressive genes and also has an antimetabolic activity, thus leading to direct cellular cytotoxicity. It has demonstrated efficacy in the treatment of myelodysplastic syndromes or AML, but is not eligible for intensive therapy. ${ }^{17}$ There is preliminary evidence of BPDCN treated with 5-azacytidine showing partial responses. ${ }^{4}$ For this reason, in our patient, we preferred to administer it as a consolidation/maintenance therapy. With azacitidine we obtained a CR by PET/CT and bone marrow aspiration. However, an early relapse occurred 3 months after discontinuation of this therapy.

\section{Conclusion}

$\mathrm{BPDCN}$ is a rare disease with an extremely poor prognosis and quick relapses after chemotherapy. ${ }^{18}$ There is no standard treatment, but better results have been obtained with intensive therapy followed by allogeneic HSCT. However, elderly patients with comorbidities are not candidates for such therapies, and new effective and low-toxicity drugs are needed.

We present preliminary evidence of the activity of lenalidomide, celecoxib, and azacitidine in an aggressive hematocutaneous BPDCN in an elderly patient achieving a CR and a 17-month OS. This information may be useful in the design of treatment approaches for elderly patients with BPDCN. However, it should be confirmed in clinical trials as well as by optimizing the induction and extending the consolidation/maintenance period to avoid early relapses after the discontinuation and improve progression-free survival.

\section{Acknowledgment}

We thank Mr Jonathan McFarland for his contribution in the revision of English language of the manuscript.

\section{Disclosure}

The authors report no conflicts of interest in this work.

\section{References}

1. Yu H, Zhang P, Yin X, et al. Human BDCA2 ${ }^{+} \mathrm{CD} 123^{+} \mathrm{CD} 56^{+}$dendritic cells (DCs) related to blastic plasmacytoid dendritic cell neoplasm represent a unique myeloid DC subset. Protein Cell. 2015;6(4): 297-306.

2. Riaz W, Zhang L, Horna P, Sokol L. Blastic plasmacytoid dendritic cell neoplasm: update on molecular biology, diagnosis, and therapy. Cancer Control. 2014;21(4):279-289. 
3. Pagano L, Valentini CG, Pulsoni A, et al. Blastic plasmacytoid dendritic cell neoplasm with leukemic presentation: an Italian multicenter study. Haematologica. 2013;98(2):239-246.

4. Laribi K, Denizon N, Ghnaya H, et al. Blastic plasmacytoid dendritic cell neoplasm: the first report of two cases treated by 5-azacytidine. Eur J Haematol. 2014;93:81-85.

5. Agliano A, Martin-padura I, Marighetti P. Therapeutic effect of lenalidomide in a novel xenograft mouse model of human blastic nk cell lymphoma/blastic plasmacytoid dendritic cell neoplasm. Clin Cancer Res. 2011;17(19):6163-6174.

6. Subhashini J, Mahipal SVK, Reddanna P. Anti-proliferative and apoptotic effects of celecoxib on human chronic myeloid leukemia in vitro. Cancer Lett. 2005;224(1):31-43.

7. Henry JY, Labarthe MC, Meyer B, Dasgupta P, Dalgleish AG, Galustian C. Enhanced cross-priming of naive $\mathrm{CD}^{+} \mathrm{T}$ cells by dendritic cells treated by the IMiDs ${ }^{\circledR}$ immunomodulatory compounds lenalidomide and pomalidomide. Immunology. 2013;139(3):377-385.

8. Ghosh N, Grunwald MR, Fasan O, Bhutani M. Expanding role of lenalidomide in hematologic malignancies. Cancer Manag Res. 2015;7: 105-119.

9. Leahy KM, Koki AT, Masferrer JL. Role of cyclooxygenases in angiogenesis. Curr Med Chem. 2000;7(314):1163-1170.

10. Pockaj BA, Basu GD, Pathangey LB, et al. Reduced T-cell and dendritic cell function is related to cyclooxygenase-2 overexpression and prostaglandin E2 secretion in patients with breast cancer. Ann Surg Oncol. 2004;11(3):328-339.

11. Dharmapuri G, Doneti R, Philip GH, Kalle AM. Celecoxib sensitizes imatinib-resistant K562 cells to imatinib by inhibiting MRP1-5, ABCA2 and ABCG2 transporters via Wnt and Ras signaling pathways. Leuk Res. 2015;39(7):696-701.
12. Zhao Y, Feng H, Jia T, et al. Antiproliferative effects of celecoxib in hep-2 cells through telomerase inhibition and induction of apoptosis. Asian Pac J Cancer Prev. 2014;15(12):4919-4923.

13. Sapienza M, Fuligni F, Agostinelli C, et al. Molecular profiling of blastic plasmacytoid dendritic cell neoplasm reveals a unique pattern and suggests selective sensitivity to NF-kB pathway inhibition. Leukemia. 2014;28(8):1606-1616.

14. Marschner N, Zaiss M. Long-term disease stabilization in a patient with castration-resistant metastatic prostate cancer by the addition of lenalidomide to low-dose dexamethasone and celecoxib. Onkologie. 2012;35(5):279-282.

15. Tsai YC, Wu CT, Hong RL. Response of refractory osteosarcoma to thalidomide and celecoxib. Lancet Oncol. 2005;6(12):997-999.

16. Prince HM, Mileshkin L, Roberts A, et al. A multicenter phase II trial of thalidomide and celecoxib for patients with relapsed and refractory multiple myeloma. Clin Cancer Res. 2005;11(15):5504-5514.

17. Pleyer L, Burgstaller S, Girschikofsky M, et al. Azacitidine in 302 patients with WHO-defined acute myeloid leukemia: results from the Austrian Azacitidine Registry of the AGMT-Study Group. Ann Hematol. 2014;93(11):1825-1838.

18. Borchiellini D, Ghibaudo N, Mounier N, et al. Blastic plasmacytoid dendritic cell neoplasm: a report of four cases and review of the literature. J Eur Acad Dermatol Venereol. 2013;27(9):1176-1181.
OncoTargets and Therapy

\section{Publish your work in this journal}

OncoTargets and Therapy is an international, peer-reviewed, open access journal focusing on the pathological basis of all cancers, potential targets for therapy and treatment protocols employed to improve the management of cancer patients. The journal also focuses on the impact of management programs and new therapeutic agents and protocols on

\section{Dovepress}

patient perspectives such as quality of life, adherence and satisfaction. The manuscript management system is completely online and includes a very quick and fair peer-review system, which is all easy to use. Visit http://www.dovepress.com/testimonials.php to read real quotes from published authors. 